\title{
Tourism Information Push System Based on Convolutional Neural Network
}

\author{
Xiaoqiang $\operatorname{Han}^{1,2, *}$,Jingwen $\mathrm{Li}^{1,2}$, Yao Hu${ }^{1,2}$, Jiao Yuan ${ }^{1,2}$, Suxian Ye ${ }^{1,2}$ \\ ${ }^{1}$ Guilin University of Technology, Guilin,Guangxi ,China \\ ${ }^{2}$ Guangxi Key Laboratory of Spatial Information and Geomatics, Guilin,Guangxi ,China
}

\begin{abstract}
In the context of the current hot tourism, personalized travel information push is the focus of major travel technology companies. For tourists, an intelligent and humane tourism push system has greatly improved tourism planning. this paper proposes a new tourism push system based on the deep learning technology of the recent hot convolutional neural network. It can satisfy the big data era by acquiring the user's image and text information for convolutional neural network analysis. The intelligent extraction of various network data and personal information and speculation of personal preferences, with a new way of self-learning to reform the current active statistics of the travel push system. The results show that the tourism information pushed by this method is ideal for satisfying the travel preferences of individual users, more humanized and intelligent, and has achieved good results.
\end{abstract}

\section{Introduction}

Online tourism has been promoted and applied with the improvement of people's living standards and the development of Internet technology. However, the problem of information overload on the Internet is becoming more and more serious due to the development of the Internet. It takes a lot of time and effort for users to obtain accurate and accurate information on the network. How to accurately find and recommend the preferred travel information for users from massive information is especially important. The increasingly popular deep learning provides a new solution to solve such problems.Deep learning adopts core algorithms such as unsupervised or semi-supervised feature learning and hierarchical feature extraction, which can avoid the process of manual selection and excessive interference, and can be used to obtain better features. ${ }^{[1]}$ Among them, the convolutional neural network (CNN) has obvious advantages over feature models in feature extraction and modeling. It interprets data by mimicking the mechanisms of the human brain, such as images, sounds, and text, while extracting abstract feature representations through more layers of network structure, enabling it to handle complex problems. It can play an ideal role in the promotion of tourist information.

\section{Convolutional neural network}

Convolutional neural networks (CNN) is a deep neural network with convolution structure. The convolution structure can reduce the amount of memory occupied by deep networks, and can also reduce the number of parameters of the network. ${ }^{[2]}$ It is also an important part of deep learning, and it is a feedforward neural network based on artificial neural network. In the 1960s, Hubel and Wiesel proposed convolutional neural networks. After decades of development, the application of convolutional neural networks has made great progress and penetrated into many areas of our lives. Since the convolutional neural network does not require complex preprocessing of the data, and it can acquire a large amount of feature information, especially in the field of pattern recognition, the convolutional neural network does not require complex preprocessing of the image, and directly uses the image as a network. The input makes the convolutional neural network have great advantages in image understanding, and it can be applied to many fields of our lives, which has gained great value.

\subsection{Typical convolutional neural network structure}

The basic structure of the convolutional neural network includes: an input layer, a convolution layer, a downsampling layer, a fully connected layer, and an output layer. ${ }^{[3]}$ Each layer has a plurality of feature maps, each of which extracts a feature of the input through a convolution filter, each feature map having a plurality of neurons.

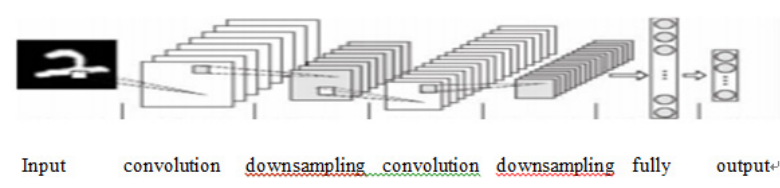

Fig. 1. Convolutional neural network example

\footnotetext{
* Corresponding author: 1269694796@qq.com
} 
Convolutional layer: The reason for using a convolutional layer is that an important feature of convolution operations is that convolution operations can enhance the original signal characteristics and reduce noise. This can greatly reduce the number of links, which greatly reduces the parameters of the network. Calculated as follows:

$$
\begin{gathered}
x_{j}^{l}=\mathrm{f}\left(u_{j}^{l}\right) \\
\mathrm{u}_{\mathrm{j}}^{l}=\sum_{i \in \mathrm{Mj}} x_{j}^{l-1} * k_{i j}^{l}+b_{j}^{l}
\end{gathered}
$$

At this level, the feature map of the upper layer is first convoluted, and then each output feature map is obtained by performing the above activation function. Downsampling layer: In this layer, the output feature map is obtained after the feature graph is calculated and calculated. The formula is as follows:

$$
\begin{gathered}
x_{j}^{l}=f\left(u_{j}^{l}\right) \\
x_{j}^{1}=\beta_{j}^{1} \operatorname{down}\left(x_{j}^{l-1}\right)+b_{j}^{1}
\end{gathered}
$$

Fully connected layer: In this layer, all 2D images are stitched into one-dimensional features as input to a fully connected network. The formula is as follows:

$$
\begin{gathered}
\mathrm{x}_{\mathrm{j}}^{l}=\mathrm{f}\left(\mathrm{u}_{\mathrm{j}}^{\mathrm{l}}\right) \\
u^{l}=w^{l} x^{l-1}+b^{l}
\end{gathered}
$$

\section{Image understanding and text analysis}

\subsection{Image understanding}

To achieve image understanding, the most important thing is the classification of images and the detection of targets in images. Image classification is also the prediction of image categories, and object detection refers to the detection of the same object or the same category of objects in the image. In image classification and object detection, general methods include methods based on Bagofwords (BOW) and methods based on Deformable Part Models (DPM). ${ }^{[4]}$ These methods have good practical effects in some fields, such as (face recognition, pedestrian monitoring, etc.), but there is still room for improvement in practical aspects. Then Krizhevsky et al. proposed new convolutional neural network structures (AlexNet), GoogLeNet and VGG. These deeper neural networks quickly overcome the problem of insufficient precision, by applying deep learning to image classification and object detection problems, to get a better result. These more advanced methods not only improve the accuracy of image understanding, but also ensure efficiency.

At the same time, with the understanding and application of the convolution neural network, there is also a processing method for the various cases of image processing. For example, for the enhancement of the complexity of mass processing images and conditions, people have proposed a variety of image understanding models, typical 3DMatch, it is a model for local point matching, and D EepContext is a computer model for understanding complex scenes. In conclusion, the adaptability of convolutional neural networks is gradually improving.
Image understanding has made unprecedented progress with people's gradual understanding and application, but the emergence of all kinds of problems is the emergence of various problems. The most prominent is the increase of data, the speed and ability to deal with data, the cross appearance of various objects in the image, and the unavoidable influence of shadow and occlusion.These are the outstanding problems to deal with.

\subsection{Analysis of emotional interest in words}

The convolutional neural network can analyze the personal emotional preferences of individual users based on various ways to obtain travel information. The core idea is to acquire emotional features. Because emotional features are the most important elements in emotional classification and recognition, we can combine emotional features with other important features, such as previous images, personal browsing records, and similar emotions. Combining a variety of features can simulate the way of thinking of the human brain, without requiring visitors to automatically fill out a series of statistical forms of travel preferences, only visitors need to open access to various applications, such as Weibo.

According to the acquired text content, the emotional features and the words in the paragraph position to construct the input matrix of the network model. Learn more about the emotional preferences of the model during the training process. Get smarter and more effective access to personal information.

The obtained input matrix is input into the convolutional neural network to perform related technical implementation, and the result can be intelligently analyzed.

For example, to obtain the information in the microblog, and then use the words as the most basic unit, each corresponding word corresponds to a multidimensional continuous vector, and the word vector matrix $E \in R_{m}{ }^{*}|V|$ of the entire data set word set can be obtained., where $\mathrm{m}$ is the vector dimension of each word, and $|\mathrm{V}|$ is the size of the collection of the data set. For a sentence of length $n s=\left\{w_{1}, w_{2}, \ldots, W_{n}\right\}$, each word wi in a sentence can be mapped to an m-dimensional vector, ie $\mathrm{e}_{\mathrm{i}} \in \mathrm{R}_{\mathrm{m}}$.

In this paper, the ordinary Howent sentiment word set is used to re-type the input sentence. By assigning the special words in the sentence to specific part-of-speech tagging, the model can make full use of the words that play an important role in the emotional classification ${ }^{[5]}$, such as positive and Negative emotion word, no

Determining words, degree adverbs, etc., should focus on learning these feature information during the training process. In the text content, sometimes not only emotional words express one's emotions, but negative words and degree adverbs must also be taken seriously. Typical examples are "like" and "dislike". After the addition of negative words, their meaning has been greatly reversed. Pay special attention to these words. Vectorized operations can be used to process these partof-speech annotations so that they can be mapped into 
multidimensional vectors. ${ }^{[6]}$ In the training process, the network model can finely adjust the components of the part of speech vector for different parts of speech, so that it can be more precise and ensure accuracy in learning. ${ }^{[7]}$

\section{Travel information push}

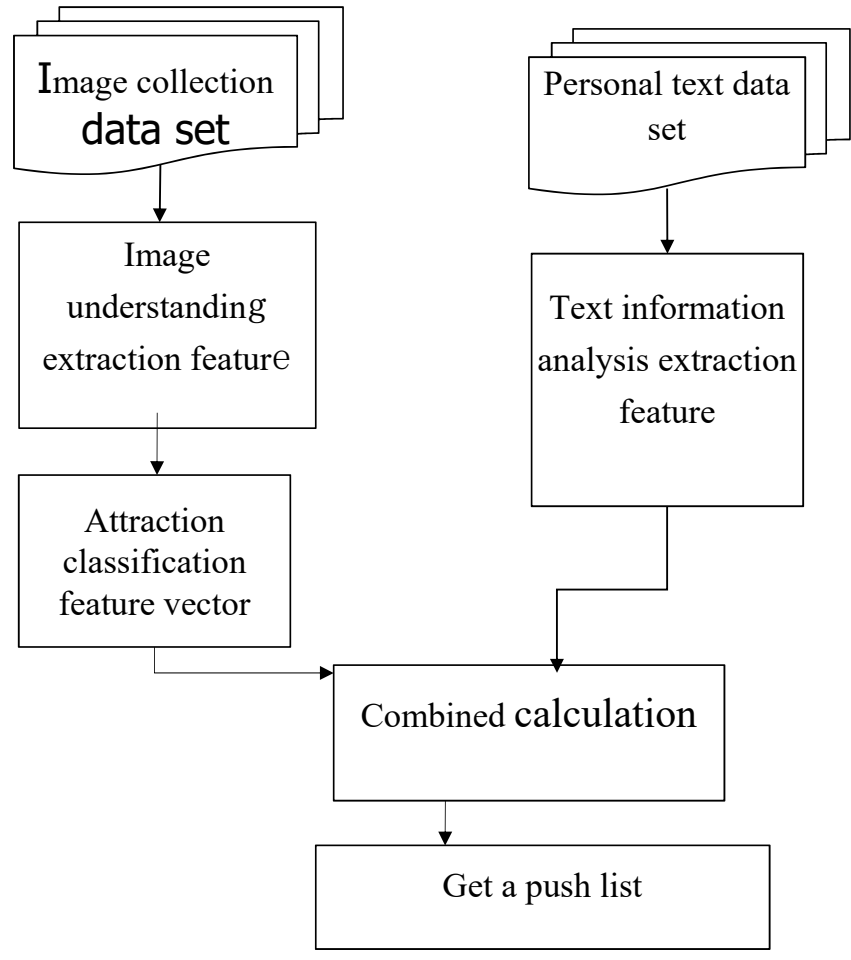

Fig. 2. Flow chart

Equations should be centred and should be numbered The tourism information push process based on convolutional neural network: firstly select the personal image set on the network application that obtains the authority as the data source, and obtain the classification summary of the individual's published scenic spot information through the convolutional neural deep learning of the image, and the threshold can be set. The way to initially determine the initial preferences, the way to judge is:

$$
T_{I}=\frac{t_{i}}{t_{l}}
$$

Where $t_{i}$ is the number of occurrences of the attraction in the $i$-th, such as historical monuments, $t_{i}$ is the total number of times of attraction of the attraction, and if it is less than the set threshold, such attraction is excluded.

While obtaining the image classification understanding, the personal emotion preferences in the text information are obtained, and the personal emotion expression can be divided into several specific numerical grades, and the personal preference spots are initially obtained by combining the images.

Finally, similar data can be found based on sentiment analysis combined with similar analysis of psychological personality on the network. As the number of deep learning increases, the increase in data volume must increase the accuracy of data speculation. Combining the extraction of images and texts and finally performing combined calculations, different weights can be set according to different data, and the calculation result is used as the basis of the preference score, and the results are sequentially arranged as a push list to the user.

At the same time, the user's historical behavior data can be collected and selected to be screened and preprocessed to divide the user's historical behavior data, which is based on the time length. The advantage of doing this is that the user's preference features can be effectively obtained. In each time window, the user's behavior features are deeply excavated, and the user's preference features in the window are depicted in detail from several different angles, thus the user's timing preference eigenvector is obtained, and the user's preference to some kind of scenic spots will become an important basis for the recommendation work. This can also be used as the basis for the important data recommended above.

\section{Conclusion}

By obtaining the rights of various Internet applications of customers, for example, micro-blog, space, and all kinds of images and words in sharing applications, and then get the text pictures of the travel mood information through screening. The collaborative information filtering algorithm based on user image and text and the similarity recommendation algorithm are introduced to help users automatically filter and filter huge tourism information data. Finally, the deep learning makes the tourism information system more intelligent and humanized. That is to meet the customer's acceptance of travel information, and does not require customers to fill in various statistical data frequently. Finally, a fast, intelligent and real-time travel information push system was realized.

It also provides a large data source that can help deal with travel information by collecting user's historical behavior data and screening and preprocessing as a large data source of recommendation information.

After using this system, the experience of tourist websites or systems is more humanized, and the information recommended by customers is more accurate. It is an effective use of the convolution neural network.

\section{Foundation items}

1. Development and Application of Intelligent Security Early Warning System for Visitors in Smart Scenic Areas and Time-Space Virtual Reconstruction Technology Item Number: 20170220

2. Research on Intelligent Indoor Positioning Method Based on WIFI and Its Application Item Number: 2018-B-02 


\section{References}

1. L. Zhao, Y. H. Song,Y. L. Zhang Parameter optimization of convolution neural network based on genetic algorithm ,China Automation Society, 7 (2017)

2. L. Chang, X.M. Deng, Convolution neural network in image understanding, Acta Automatica Sinica , 42 (2016)

3. Y. D. Li, Z.B. Hao, A review of convolution neural networks, Journal of Computer Applications , 36(2016)

4. FelzenszwalbP, McAllesterD, Multiscale Deformablepartmodel,Proceedingsofthe2008IEEEC onferenceonComputerVisionandPatternRecognition. Anchorage, (2008)

5. K. Chen, B. Liang,W .D. Ke,B .Xu,G. C. Zeng ,Sentiment analysis of chinese Micro-blog based on multi-channel convolutional neural network , Journal of Computer Research and Development, 55 (2018)

6. Y. Wang,H. Y. Zhou, Network traffic classification method basing on CNN, Journal on Communications,39(2018)

7. F.Y.Zhou,L.P.Jin,Review of Convolutional Neural Network, Chinese Journal of Computers, 10(2017) 